\title{
Injured neurons pump up the volume
}
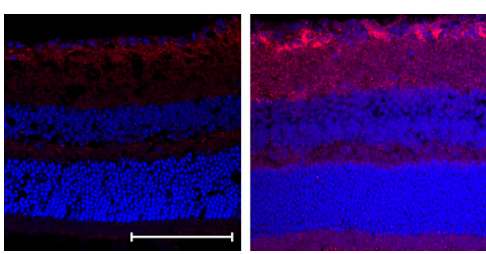

DLK (magenta) is scarce in an uninjured retina (left), but prevalent in a retina three days after injury (right).

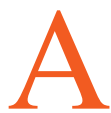

wounded axon sends out a long-distance distress signal. HuntworkRodriguez et al. uncovered a positive feedback loop that might amplify the alarm and help it spread along the axon.

A damaged axon dispatches an alert to the cell body, which fires up genes thatdepending on the neuron's location-trigger healing or cause the axon to degenerate and the cell to die. Key proteins in this response are the c-Jun N-terminal kinases (JNKs), which spur axon deterioration and cell death. Another important protein is the dual leucine zipper-bearing kinase (DLK), which senses axon injury and ramps up JNK activity. Researchers think that the molecular distress call travels along the axon when JNKs hop aboard motor proteins such as dynein.

\section{Drebrin shows self-restraint}

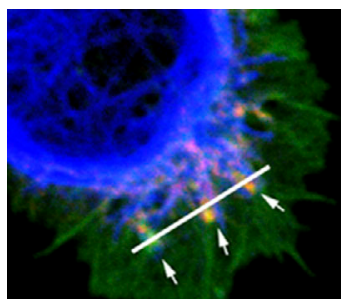

Drebrin (red) and F-actin (green) convene at the tip of a neuron that is just beginning to grow.

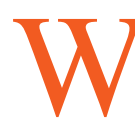

orth et al. reveal that the protein drebrin, which is crucial for neuron development and memory, controls its own activity by folding over on itself.

Drebrin bolsters the dentritic spines that store memories, and it vanishes from these spines in people who have Alzheimer's disease and other memory-robbing conditions. During development, drebrin performs other tasks, promoting the extension of neurites and helping direct growing neurons in the right direction. At the elongating tip of a neuron, drebrin links microtubules to actin filaments, thus possibly strengthening the tip and ensuring the delivery of necessary materials. Drebrin can also drive the formation of F-actin bundles, which brace cellular extensions. But researchers don't understand how the protein interacts with actin.
Huntwork-Rodriguez et al. uncovered the system's volume knob. They sliced the axons of the optic nerve in mice and found that DLK levels rapidly shot up at the site of the damage. Within three days, the DLK surge had spread to the cell bodies of the neurons. However, the cells weren't making more DLK; they were destroying less. The researchers discovered that stress causes neurons to reduce their ubiquitylation of DLK, thus sparing the protein from the proteasome.

Phosphorylating DLK at several sites protects it against ubiquitylation. JNKs perform this phosphorylation, setting up a positive feedback loop. The researchers think that cells normally use ubiquitylation to keep DLK levels under tight control. But an injury switches on DLK locally, which in turn flips on JNKs that phosphorylate DLK and prevent ubiquitylation, leading to increased DLK levels and more JNK activation. Without this boost, the response can't spread toward the cell body.

Huntwork-Rodriguez, S., et al. 2013. J. Cell Biol. http://dx.doi.org/10.1083/ jcb.201303066.

Worth et al. investigated the interaction by running a computer analysis of drebrin's amino acid sequence, which revealed five domains in the molecule. The researchers engineered kidney cells to manufacture drebrin variants that were missing different sections and then tested the molecules' abilities to latch onto actin and spur filopodia to sprout, a sign that they could bundle actin. The team found that two domains-a coiled-coil domain and a helical domain-worked together to bundle actin. However, in vitro experiments on recombinant drebrin made by bacteria found that it was a feeble actin bundler.

The explanation for this apparent contradiction, Worth et al. discovered, is that drebrin inhibits itself. The molecule doubles over when two of the non-actin-binding domains interconnect, obstructing the coiled-coil domain. Cells can remove this inhibition by enlisting the protein $\mathrm{Cdk} 5$, which affixes a phosphate group near the coiled-coil domain and exposes the domain to actin. Local activation of Cdk5 could couple microtubules to F-actin at a specific site and spur growth in that direction.

Worth, D.C., et al. 2013. J. Cell Biol. http://dx.doi.org/10.1083/ jcb.201303005. 